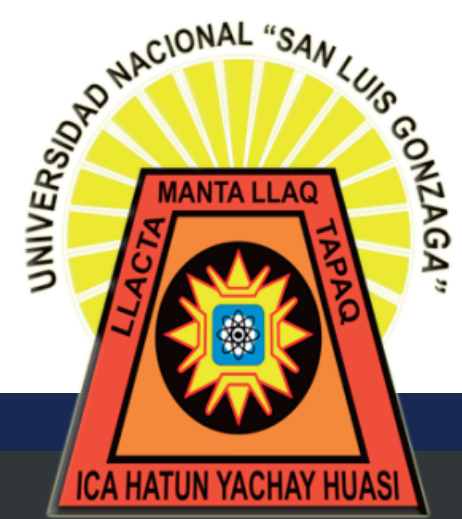

p-ISSN 2223-2893

e-ISSN 2225-6989

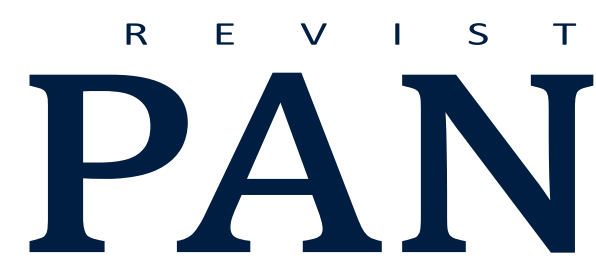

UNIVERSIDAD NACIONAL SAN LUIS GONZAGA. ICA, PERÚ
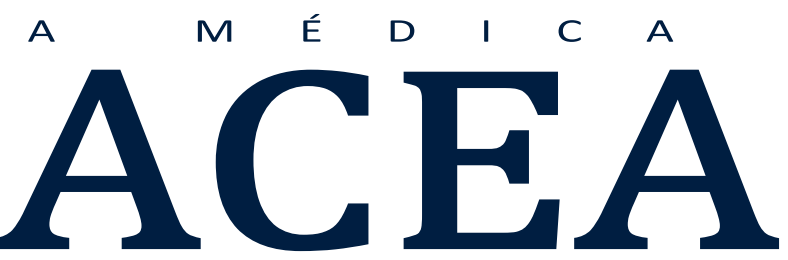

FACULTAD DE MEDICINA HUMANA "DANIEL ALCIDES CARRIÓN"

ARTÍCULO ORIGINAL:

VOLUMEN 10 NÚMERO 1

PUBLICACION CUATRIMESTRAL

ENERO - ABRIL

2021

\title{
VALORES ÉTICOS Y ACTITUDES DE LOS DOCENTES DE MEDICINA EN UNA UNIVERSIDAD PÚBLICA, ICA 2019.
}

\section{AUTORES:}

LÉON LLACSA SOTO

LUZ FIGARI SÁNCHEZ

INDEXADA EN:
$\underset{\text { catálogo }}{\operatorname{latin}}$
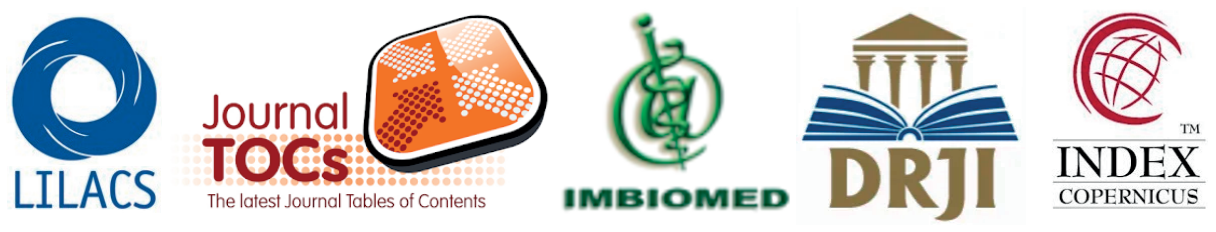
Google

\section{REVISTAS.UNICA.EDU.PE}




\title{
VALORES ÉTICOS Y ACTITUDES DE LOS DOCENTES DE MEDICINA EN UNA UNIVERSIDAD PÚBLICA, ICA 2019.
}

\author{
Llacsa-Soto Léon', Figari-Sánchez Luz². \\ 1. Profesor Principal Facultad de Medicina - UNSLG. Magister en \\ Bioética y Biojurídica - USAT. \\ ORCID: orcid.org/0000-0002-9667-5664
}
2. Profesora Principal Facultad de Medicina, Doctora en Medicina - UNSLG. ORCID: https://orcid.org/0000-0002-9958-125X.

DOI: $10.35563 /$ rmp.v10i1.405

\section{Correspondencia:}

Autor corresponsal: León

Llacsa Soto.

Dirección: naranjos E - 13

Urb. San Luis, Ica, Perú.

Teléfono: 056622928

Correo electrónico:

leonllacsa7@gmail.com

Contribuciones de autoría: LLSL y FSL: participó en el diseño del estudio, análisis, redacción, revisión y aprobación final del manuscrito.

Conflicto de intereses: no existen conflictos de intereses del autor o autores de orden económico, institucional, laboral o personal.

Financiamiento:

Autofinanciado.

\section{RESUMEN}

Objetivo: Determinar qué valores y actitudes identifican los docentes de la Facultad de Medicina. Materiales y métodos: un estudio por encuesta basada en el estudio de Prieto a 44 docentes con interrogantes sobre conceptos de ética, deontología y sobre valores personales y sociales. Resultados: Conceptúan a la ética como moral (52\%), como filosofía (4.5\%), a la deontología la definen como valores (56\%) y como deberes (15\%). Respecto a Valores personales, a la interrogante ¿qué es lo más importante en la vida?, señalan: la salud (38\%), la familia (38\%), realización personal (5\%). Respecto a Valores ético sociales, a la interrogante ¿qué quiere Ud. para este mundo?, responden: Justicia social (50\%), la paz (29\%), respeto al ambiente (11\%). Respecto a Valores éticos morales, a la interrogante ¿cómo hay que comportarse con los otros?, señalan: con honestidad (45\%), con respeto (25\%), con solidaridad (5\%). Definen a la actitud como: disposición (60\%), conducta (36\%); Señalan a las cuatro actitudes a priorizar en la docencia: motivación (88\%), abierto al cambio (43\%), disposición a ayudar (38\%), diálogo (34\%). Respecto a la enseñanza de la ética, a la interrogante ¿quiénes deben ser docentes en ética? Señalan: con alguna capacitación (52\%), maestría en ética (38\%). Conclusiones: los docentes no muestran claridad conceptual de ética ni de los valores personales base de los sociales. Sí definen las actitudes prioritarias en la docencia. La mayoría reconoce la necesidad de capacitarse en ética. Se sugiere efectivizar la mejora de sus competencias ético morales.

Palabras clave: Ética, valores, actitudes y docencia universitaria.

\section{ABSTRACT}

Objective: To determine which values and attitudes are identified by teachers of the School of Medicine. Materials and methods: a survey study based on Prieto's study to 44 teachers with questions on concepts of ethics, deontology and personal and social values. Results: they define ethics as moral (52\%), as philosophy (4.5\%), deontology as values (56\%) and as duties (15\%). Regarding personal values, to the question "What is the most important thing in life?", they indicate: health (38\%), family (38\%), personal fulfillment (5\%). With respect to social ethical values, to the question "What do you want for this world?", they respond: social justice (50\%), peace (29\%), respect for the environment (11\%). Regarding moral ethical values, to the question "How should we behave with others?", they indicate: with honesty (45\%), with respect (25\%), with solidarity (5\%). They define attitude as: disposition (60\%), conduct (36\%); they point out the four attitudes to be prioritized in teaching: motivation (88\%), open to change (43\%), willingness to help (38\%), dialogue (34\%). Regarding the teaching of ethics, to the question: who should be teachers of ethics? They indicate: with some training (52\%), master's degree in ethics (38\%). Conclusions: teachers do not show conceptual clarity of ethics or of personal values as a basis for social values. They do define priority attitudes in teaching. Most of them recognize the need for training in ethics. It is suggested to improve their ethical and moral competencies.

Keywords: Ethics, values, attitudes and university teaching. 


\section{INTRODUCCIÓN}

La ética como rama de la filosofía que define la conducta ideal del ser humano caracteriza al ethos, como forma de vida o modo de ser en el sentido más profundo, se manifiesta a través del comportamiento moral y ésta se orienta por los principios y valores, para determinar las actitudes y la identidad moral, que forman parte del comportamiento humano en los diversos sectores de la sociedad, como personas, profesionales o en un colectivo (1)

Como toda actividad humana, la labor docente está sujeta a deberes, como parte de su dimensión moral junto al científico técnico, integrados en el marco formativo. Su responsabilidad no se limita sólo a transmitir conocimientos y técnicas, sino a establecer y a aplicar los fundamentos de la vida moral, valores, virtudes y actitudes.

La educación es la fuente de valores de la persona, donde aprende grandes virtudes que garantizan su futuro. Valor significa fuerza, energía, potencia de un ser, si bien su noción es referida de la economía para indicar utilidad o precio, su estimación es determinada siempre por el sujeto, el valor no es, pero vale, los expresamos en la convicción o aspiración razonada de lo que es bueno o malo, y una vez interiorizado, se convierten en normas de conducta individual y sociales. (2)

Los Estudios relacionados muestran la importancia de la actitud del docente en asumir su identidad profesional (1), con los valores a fomentar con el ejemplo, como factor motivacional de la personalidad a la vez de regulador de sus acciones, y su responsabilidad de la educación en valores $(2,3,5)$

La actividad académica que incluye a la labor docente en la universidad, prioriza el desarrollo de las capacidades cognitivo procedimentales con poco o ningún énfasis en el refuerzo o motivación de la dimensión moral de los actores del proceso enseñanza aprendizaje, falencia que se ha hecho casi tradicional y se mantiene aún, a pesar que se viene estableciendo cambios importantes después de puesta en vigencia de la nueva ley universitaria desde 2014.

Estudios nacionales enfatizan la necesidad de desarrollar la ética profesional y deontológica del docente, e iniciar su formación en los estudiantes de pregrado, así como de la normatividad a la que estarán sujetos en su práctica profesional futura. Así como de situar a los valores como pilares del comportamiento, estándares válidos de calidad de personas o grupos y necesidades más significativas para el desarrollo social, en el plano de conciencia moral y social, $(6,14)$.

La crisis ético moral y social mundial se evidencia más a nivel de Latinoamérica y en el marco del sistema educativo se expresa en los resultados que muestran en su conducta los profesionales egresados de las diferentes áreas, que luego conforman la conducción de las instituciones públicas y privadas; por ello es importante promover estudios de exploración y de reconocimiento en el marco de la dimensión moral del docente universitario.

La formación médica hace más énfasis en los aspectos biológicos técnicos y poco en lo psicosocial la condición de ser médicos y a la vez docentes determina por los fines de la medicina desarrollar una ética profesional del docente a plenitud por el diálogo y ejemplo, induzca y motive a las acciones con sentido, valores y virtudes que se expresen en las actitudes y comportamientos cada vez más coherentes y significativos durante el proceso formativo que aseguren la aplicación y vigencia de los principios éticos fundamentales. $(3,7,10)$.

En ese contexto, el objetivo del presente estudio es determinar qué valores y actitudes identifican los docentes de la Facultad de Medicina en la práctica formativa, considerando los graves episodios periódicos de inconductas morales, o indiferencia latente en unas facultades más que en otras, ahora en el marco de vigencia de una nueva Ley Universitaria y ante un proceso de Licenciamiento al que deben acogerse..

\section{MATERIALES Y MÉTODOS}

Es un estudio transversal, descriptivo a través de una encuesta cuestionario semi estructurado que incluye 10 interrogantes relativos al tema, en base al estudio de Prieto. A y col. ( 7 ) y aplicados al azar a una muestra de 44 docentes de un total de 80 de la Facultad de Medicina de la Universidad San Luis Gonzaga de Ica, el cuestionario contiene: datos generales y las interrogantes sobre: conceptos de ética, deontología, y en relación a los valores personales, sociales y morales: qué es lo importante en la vida?, qué quiere para el mundo?, cómo debe comportarse con otras personas?, qué tener para competir en la vida?, luego su concepto de actitud, e identificar 4 actitudes a priorizar en la práctica docente, y finalmente cómo debe capacitarse en ética?, y quiénes deben ser los docentes de ética? Así mismo el proyecto del trabajo se sometió consideración del comité de ética de la Facultad de Medicina sin observaciones.

\section{RESULTADOS}

Los datos generales de los encuestados: indican que son de género masculino, mayores de 35 años y de categoría Principal en $\%$ de 60 - 65, trabajan en el área de clínicas médico quirúrgicas en el $70 \%$, y tienen grado de maestría el $95 \%$, de doctor $5 \%$, en la Universidad.

En relación a los resultados de las interrogantes de la encuesta se grafican como sigue:

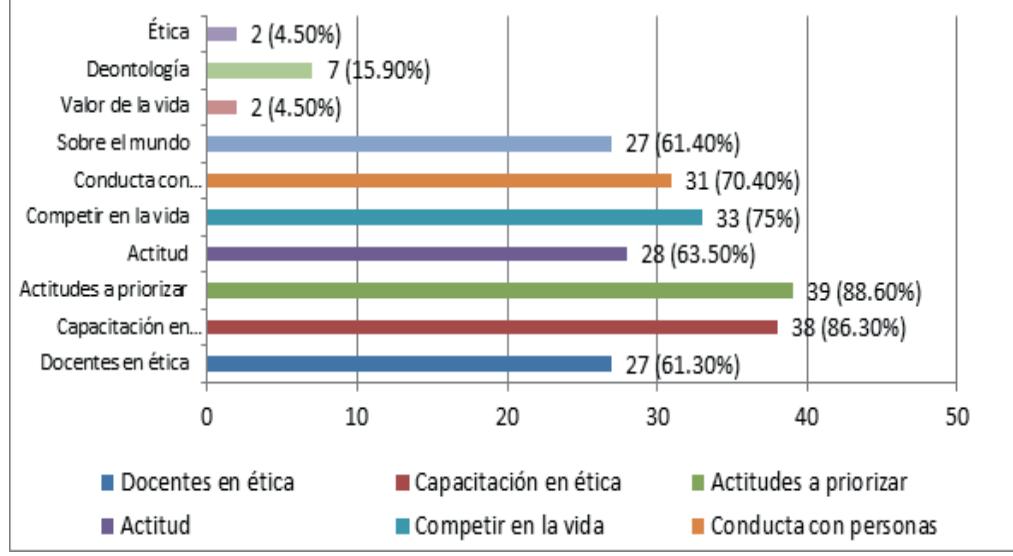

Gráfico 1. Respuestas a los temas del cuestionario 


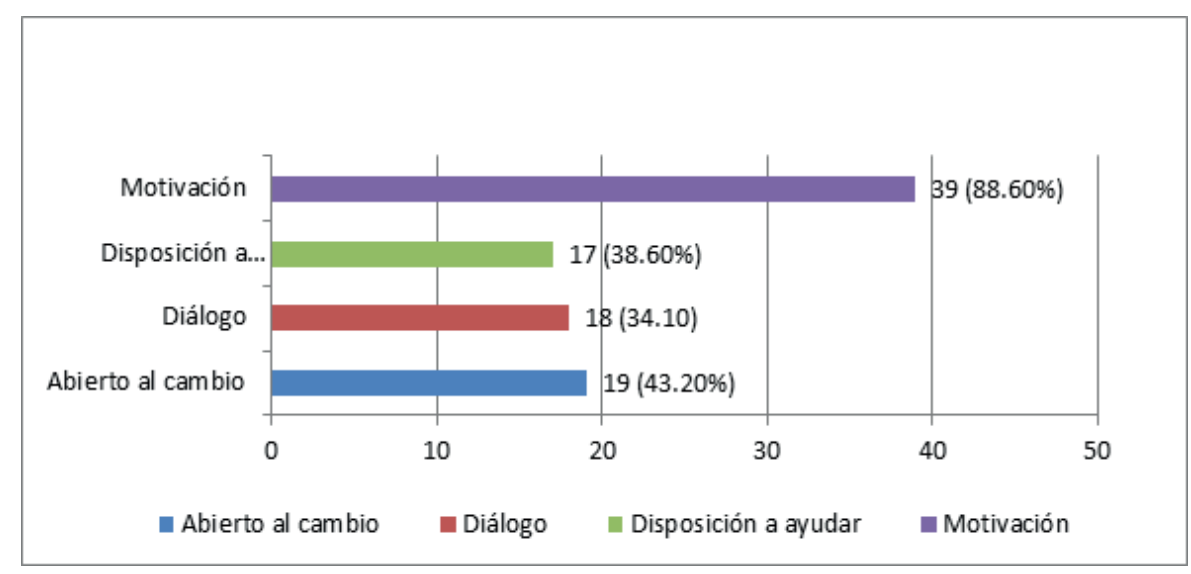

Gráfico 2. Refiera cuatro actitudes a priorizar en la práctica Docente

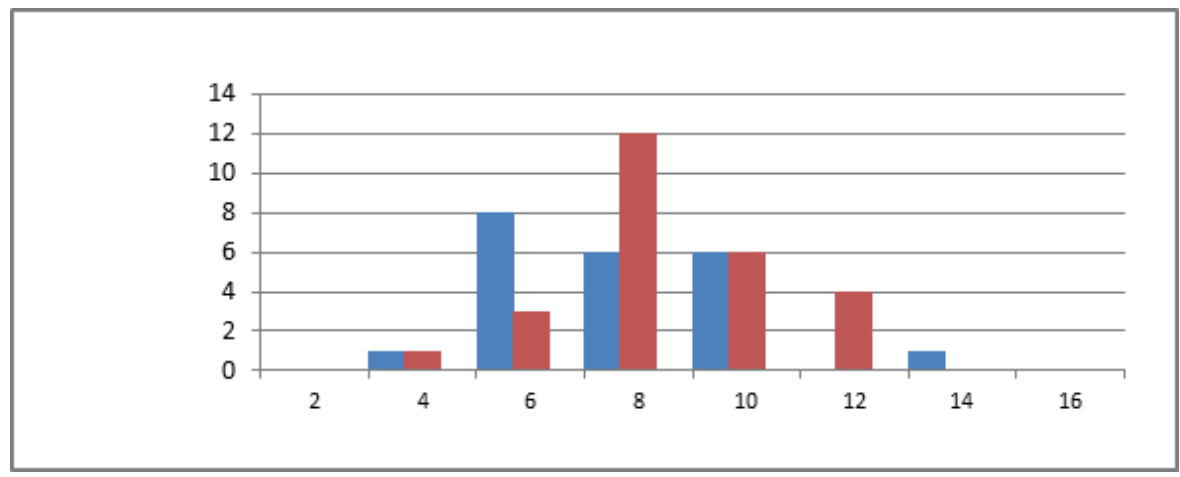

Gráfico 3. Distribución de las puntuaciones totales

\section{DISCUSIÓN}

Dada la importancia de la ética en la educación universitaria para garantizar su calidad, obliga ella a la institución a constituirse en un espacio óptimo de aprendizaje, no sólo de carácter profesional y cultural, sino también de carácter humano y por ende ético, moral y social. En ésa orientación los datos de la encuesta cuestionario muestran un grupo de docentes en su mayoría masculino, con categoría de principal y con el grado de maestría, ellos conceptúan a la ética aún como moral ( $52 \%$ ) y como disciplina ( $29 \%$ ) y como filosofía (4.5\%); a la deontología como valores ( $56 \%$ ) y como deberes ( $15 \%$ ), cuando están ya consensuados su concepto como filosofía y deberes respectivamente como lo reporta Izarra .D (4, 5$)$; en relación a los valores personales a través de la interrogante, ¿qué es lo más importante en la vida?: señalan a la salud ( $38 \%$ ) y a la familia ( $38 \%$ ), a la realización personal, sólo ( $4.5 \%$ ); en relación a los valores ético sociales: por la interrogante, ¿qué quiere Ud. para éste mundo?: justicia social ( $50 \%$ ), paz ( $29 \%$ ) y respeto al ambiente sólo ( $11 \%$ ), Sobrevivencia $3 \%$; considerando que el rol del docente es el de orientación en la construcción de la personalidad del alumno a través del enfoque de su realidad social, la realización personal y la justicia social respectivamente, son respuestas más aceptadas en otros estudios como Orna. O (6), Gonzales. M (13); en relación a los valores ético morales o de conducta, señalan a la honestidad ( $45 \%$ ), respeto ( $25 \%$ ) y a la responsabilidad y solidaridad cada uno con solo ( $4.5 \%$ ), donde la mayoría de autores señalan a la responsabilidad, el respeto y la honestidad, Prieto. A, Hirsch y A, Serna. A $(7,8,12)$; para los valores de competencia por la interrogante, ¿qué hay que tener para competir en la vida?, responden educación ( $54 \%$ ), inteligencia ( $20 \%$ ), trabajo ( $13 \%$ ), se muestran respuestas coincidentes en Muñoz. E y Crisol. E $(9,10)$; volviendo a lo conceptual, definen la actitud como disposición ( $60 \%$ ), como conducta ( $36 \%$ ) y señalan como las cuatro actitudes a priorizar en la práctica docente, a la motivación ( $88 \%$ ), abierto al cambio ( $43 \%$ ), disposición a ayudar solo(38 \% ) y al diálogo solo ( $34 \%$ ), sobre otras alternativas que tenían: como la empatía, el ser autocrítico y con equidad, con variación en el orden o como valores ético sociales como se mencionan también en Prieto y $\mathrm{A}$, Mauri. M $(7,11)$; y a la pregunta sobre el valor de la ética, ¿cómo debe ser la capacitación en ética?, responden como obligatoria ( $72 \%$ ), opcional aún el ( $13 \%$ ) y a la pregunta ¿quiénes deben ser docentes en ética? con alguna capacitación (52\%), con maestría en ética (38 \%) y todos ( $9 \%$ ), por lo menos la mayoría coincide en la necesidad de capacitación en ética y quiénes deben dictarlo.

Por el carácter de medición desde la pedagogía y a pesar de la relatividad de la interpretación de resultados por el tamaño de la muestra, el carácter indirecto de las interrogantes y otros factores, es posible dentro de un marco de evaluación cuantitativa aproximarlo al sistema vigesimal, y el promedio de la distribución de las puntuaciones que se encuentra es de 9.4 ( cuadro 12 ), que sería compatible interpretar con una caracterización que a modo de conclusiones podría ser referido como sigue:

De los resultados se puede afirmar que los docentes no muestran aún claridad de expresión de los términos de ética y deontología; no identifican suficientemente los valores personales y sociales más importantes en el proceso educativo; sí se define la actitud por los encuestados y a la vez señalan las 
actitudes a priorizar en el proceso, y sí se reconoce por la mayoría, la necesidad de tener capacitación suficiente en ética.

Luego es pertinente asumir lo referido en el marco de la educación en valores dentro del proceso del sistema curricular integrado y orientado a complementar la formación y desarrollo de la personalidad de los estudiantes como parte de sus competencias ético sociales a lograr. $(13,15)$

\section{CONCLUSIONES:}

Los docentes no muestran claridad conceptual sobre la ética más que la deontología, no identifican los valores personales como la base de los valores sociales, sin embargo, reconocen algunas actitudes a priorizar durante la práctica docente.

Lo que expresaría que no existe aún el interés personal e institucional suficiente, para establecer las medidas que posibiliten lograr un mejor nivel de competencia ético social de los principales actores del proceso educativo en la universidad. Se sugiere por ello a los niveles de gestión, considerar su prioridad para adoptar las estrategias que mejoren las competencias ético sociales. 


\section{REFERENCIAS BIBLIOGRÁFICAS}

1. Ibarra G. Ética e identidad docente, Simposio internacional, Barcelona 21- 22 nov 2013

2. Molina Luzcarin, Pérez Pellín Siulbel, Suárez Auxifrantys, Rodríguez G William A. La importancia de formar en valores en la educación superior. Acta odontol. venez [Internet]. 2008;46(1): 41-51

3. Soto, D. El profesor universitario de América latina: hacia una responsabilidad ética, científica y social, gpo. Invest HISULA, UPT de Colombia, Rhela, 2009; 13:166-168.

4. Izarra, D. Ética en la formación docente, Univ. Pedagógica experimental, Institu. Mejoramiento profesional, Táchira, Ven. 2006

5. Cobo, J.M. Universidad y ética profesional, Univ. Pontificia Comillas, Madrid, 2004

6. Orna, O Ética y deontología del docente universitario, edit. Univ. Ricardo Palma, Lima, 2006

7. Prieto, A. Zambrano, Rivera, Y Principios éticos y valores en la formación del docente universitario, Univ Zula, Ven 2014

8. Hirsch, A Ética profesional y profesores de pos grado en México, Fractal, Rev. de Psicología, 2010;22:(1).

9. Muñoz, E. Ética y valores en la investigación y en la docencia universitaria, Univ. Internacional de Andalucía, 2008

10. Crisol, M. y Romero, M.A. "Práctica docente versus ética docente". Hacia la mejora de la práctica docente a partir de la ética profesional. Journal for Educators, Teachers and Trainers. 2014;5(2), pp. 23-35.

11. Mauri, M. La enseñanza universitaria de la ética, en Desarrollo moral y metafilosofía, USA, 2002

12. Serna, A. Luna, E. Valores y competencias para el ejercicio de la docencia de pos grado, Sinéctica. 2011;37(2).

13. Gonzales, M. E. Ética y bioética en la educación médica, Rev. Ven, Historia de la medicina, 2017;66(1-2).

14. Vera M, Cornejo R, Rivas L, Calizaya J, Zamora O, García J. Significado de los valores en docentes y Estudiantes de enfermería, UNMSM, 2014

15. Gonzáles R, Cardentey J. Educación en valores de estudiantes universitarios. Rev. bibliográfica, Rev. Humanidades Médicas, 2016; 6:(1):161-174. 